\title{
Stellar differential rotation and inclination angle from spectro-interferometry
}

\author{
A. Domiciano de Souza ${ }^{1,2,3}$, J. Zorec ${ }^{4}$, S. Jankov ${ }^{1,2,5}$, F. Vakili ${ }^{1,2}$, L. Abe ${ }^{1}$, and E. Janot-Pacheco ${ }^{6}$ \\ ${ }^{1}$ Laboratoire Universitaire d'Astrophysique de Nice (LUAN) - UMR 6525, Univ. de Nice-Sophia Antipolis, Parc Valrose, \\ 06108 Nice Cedex 02, France \\ 2 Observatoire de la Côte d'Azur (OCA), Dépt. Fresnel, UMR 6528, Av. Nicolas Copernic, 06130 Grasse, France \\ 3 Max-Planck-Institut für Radioastronomie, Auf dem Hügel 69, 53121 Bonn, Germany \\ 4 Institut d'Astrophysique de Paris (IAP), 98bis, Bd Arago, 75014 Paris, France \\ 5 Astronomical Observatory Belgrade, MNTRS 1940, Volgina 7, 11050 Beograd, Yugoslavia \\ ${ }^{6}$ Instituto de Astronomia, Geofísica e Ciências Atmosféricas da Universidade de São Paulo (IAG-USP), CP 9638, \\ 01065-970 São Paulo (SP), Brazil
}

Received 5 May 2003 / Accepted 15 January 2004

\begin{abstract}
Differential interferometry (DI) is a technique that combines high spectral resolution with high spatial resolution. It is particularly suited to probe mechanisms that induce chromatic signatures, such as large scale mass motions. We thus investigate the use of DI combined with the Fourier transform method to study the stellar surface differential rotation. We show that, compared to spectroscopy, DI has the double advantage of allowing the use of all available Fourier frequencies as well as of disentangling the stellar differential rotation rate and the inclination angle. We derive some reference analytical expressions of DI observables. It is shown that the foreseen instrumental performances of the spectro-interferometer VLTI/AMBER are high enough to study the stellar differential rotation.
\end{abstract}

Key words. techniques: high angular resolution - techniques: interferometric - techniques: spectroscopic methods: data analysis - stars: rotation

\section{Introduction}

On the stellar surface, phenomena such as convection, meridional circulation, turbulence, rotationally induced global and local hydrodynamical and magneto-hydrodynamical instabilities, non-radial pulsations and zonal flows. Neglecting smallscale motions of material, which redistribute the effective mean atomic-line absorption coefficient, the presence of large scale velocity fields in the external layers of the star lead to measurable Doppler line broadenings (Huang \& Struve 1953). These large-scale motions can significantly distort, either deepening or shallowing, the spectral line profiles in comparison with those predicted by classical models of uniformly rotating stars. Departures from uniform rotation were analyzed in the past by many authors. For instance see Gray (1982), Stoeckley \& Buscombe (1987), Gies \& Kullavanijaya (1988), Cranmer \& Collins (1993), Zorec (1994), Jankov et al. (2001), among others.

A powerful technique for measuring subtle features in stellar spectral lines is the Fourier transform (FT) method (e.g.

Send offprint requests to: A. Domiciano de Souza, e-mail: Armando.Domiciano@obs-azur.fr
Gray 1973, 1975). Since the positions of the FT zeroes are insensitive to the characteristics of the local line profiles, they make it possible to infer several stellar parameters such as the projected rotational velocity and the differential rotation. When it comes to differential rotation, several authors have shown that it cannot be disentangled from the stellar aspect angle (inclination) if only spectroscopic data are used (Gray 1977; Bruning 1981; Reiners \& Schmitt 2002, 2003).

It is then important to scrutinize the possibility of detecting and measuring large-scale motions in stellar surfaces using new observing techniques. Among them, the use of differential interferometry (DI; Beckers 1982; Labeyrie 1970) together with the FT method, can be suitable (Vakili et al. 1997; Jankov et al. 2001). DI combines high spectral and spatial (or angular) resolution data.

The advantages of combining FT of DI data to determine the stellar angular diameter were put forward by Chelli \& Petrov (1995a,b) and Petrov et al. (1995). They showed that by this technique the influence of the local profile is removed in all Fourier frequencies and that consistent results can be obtained, even though the angular resolution is not very high. Following these findings, we analyse here the performances of DI 
combined with FT to study the stellar surface differential rotation and the way of disentangling the inclination angle from it.

An introductory description of the DI theory is given in Sect. 2, where we present the concepts of differential phase and photocenter. In Sect. 4.2 we introduce the test differential rotation law adopted in this work, show examples of radial velocity maps and illustrate the differential rotation signatures on DI observables (spectra and photocenters). In Sect. 4 we explore a wide range of the parameter space in order to inquire into the behavior of spectra and photocenters, as well as into their FT. In this section we also show that the stellar inclination and the differential rotation rate can be disentangled by DI observations combined with the FT method. In Appendix A we develop the corresponding mathematical grounds showing that, for arbitrary surface velocity fields and uniform local line profile, spectra and photocenters across the line profiles can be expressed as convolutions. In Sect. 5 we point out a possible self-consistent parameter determination and discuss shortly some instrumental requirements for DI observations of differentially rotating stars. The summary and the main conclusions are gathered in Sect. 6. The needed reference analytical expressions of DI observables and their FT for a limb darkened uniformly rotating star are given in Appendix B.

\section{Basics on differential interferometry}

\subsection{Differential phase in the interferometric fringes}

The image $\iota(\boldsymbol{r}, \lambda)$ of a target obtained by an astronomical instrument at the wavelength $\lambda$ is defined by the following convolution:

$\iota(\boldsymbol{r}, \lambda)=I(\boldsymbol{r}, \lambda) * A(\boldsymbol{r}, \lambda)$

where the vector $\boldsymbol{r}$ is the position on the sky, $I(\boldsymbol{r}, \lambda)$ is brightness distribution of the object projected onto the sky, called "intensity map" and $A(\boldsymbol{r}, \lambda)$ is the point spread function (PSF) due to the instrument and the terrestrial atmosphere.

The time-averaged cross spectrum $\mathcal{W}$ between two images recorded simultaneously in a narrow spectral channel centered at $\lambda$ and a wider reference channel centered at $\lambda_{r}$ is:

$\mathcal{W}\left(\boldsymbol{u}, \lambda, \lambda_{r}\right)=\left\langle\tilde{I}(\boldsymbol{u}, \lambda) \tilde{A}(\boldsymbol{u}, \lambda) \tilde{I}^{*}\left(\boldsymbol{u}, \lambda_{r}\right) \tilde{A}^{*}\left(\boldsymbol{u}, \lambda_{r}\right)\right\rangle$

where \langle\rangle represents the ensemble average operation, $\tilde{I}$ and $\tilde{A}$ are the Fourier transforms of $I$ and $A$ respectively, and the symbol $*$ represents the complex conjugate quantity. The vector $\boldsymbol{u}$ is the spatial frequency associated with $\boldsymbol{r}$. In this paper we consider the use of DI combined with long baseline interferometry, so that $\boldsymbol{u}$ is given by the vector $\boldsymbol{B}_{\text {proj }} / \lambda$, where the modulus $B_{\text {proj }}$ is the sky-projected baseline that is considered much larger than the individual telescope aperture.

If $\left|\lambda-\lambda_{r}\right|$ is small enough $(\sim 10 \mathrm{~nm})$, Eq. (2) can be simplified assuming that the PSF is wavelength-independent inside this spectral range:

$$
\begin{aligned}
\mathcal{W}\left(\boldsymbol{u}, \lambda, \lambda_{r}\right) & =\tilde{I}(\boldsymbol{u}, \lambda) \tilde{I}^{*}\left(\boldsymbol{u}, \lambda_{r}\right)\left\langle|\tilde{A}(\boldsymbol{u})|^{2}\right\rangle \\
& =|\mathcal{W}| \mathrm{e}^{\phi\left(\boldsymbol{u}, \lambda, \lambda_{r}\right)}
\end{aligned}
$$

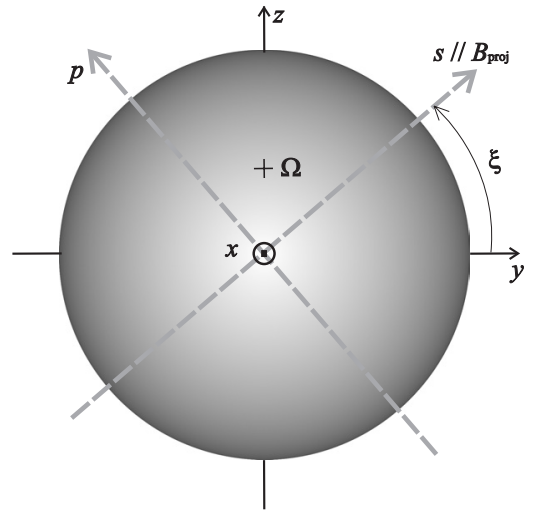

Fig. 1. Adopted reference system for a rotating star. The cross indicates the point where the rotation axis crosses the stellar surface. The $x$ axis points towards the observer and the $(y, z)$ plane describes the sky plane. The rotation axis forms an angle $i$ (not shown in the figure) with the observer direction and its projection onto the sky is parallel to the $z$ axis. The sky-projected interferometer baseline $\boldsymbol{B}_{\text {proj }}$ forms an angle $\xi$ with the $y$ axis and defines a new sky-projected coordinate system $(s, p)$, where $s$ (spectrograph slit direction) is parallel to $\boldsymbol{B}_{\text {proj }}$. All spatial coordinates are measured in angular units.

where the instrumental transfer function $\left\langle|\tilde{A}(\boldsymbol{u})|^{2}\right\rangle$ is real and positive. Note the interesting property of the phase $\phi$ of $\mathcal{W}$ (differential phase), because it is independent of the instrument and contains direct information about the target (Chelli \& Petrov 1995a,b).

\subsection{Line spectra and photocenters}

Most stars have angular diameters $\oslash$ much smaller than the highest spatial resolution attainable by presently operating interferometers, so that $\boldsymbol{u} . \boldsymbol{r} \ll 1$. Even in this case, however, it is still possible to obtain high angular resolution information using DI. This becomes clear by considering only the zero and first order terms of a Maclaurin expansion of $\tilde{I}(\boldsymbol{u}, \lambda)$ (Jankov et al. 2001). The differential phase can then be written as:

$\phi\left(\boldsymbol{u}, \lambda, \lambda_{r}\right)=-2 \pi \boldsymbol{u} \cdot\left[\boldsymbol{\epsilon}(\lambda)-\boldsymbol{\epsilon}\left(\lambda_{r}\right)\right]$.

The vector quantities $\boldsymbol{\epsilon}(\lambda)$ and $\boldsymbol{\epsilon}\left(\lambda_{r}\right)$ are called photocenters for $\lambda$ and $\lambda_{r}$ respectively. A photocenter is the center of gravity of the intensity map $I$ (photometric barycenter) measured in angular units. The usual procedure in DI is to take $\lambda_{r}$ on the adjacent continuum of a spectral line and to put $\epsilon\left(\lambda_{r}\right)=0$, which does not imply any loss of generality. Adopting the cartesian reference frame depicted in Fig. 1, we can write:

$\boldsymbol{\epsilon}(\lambda)=\epsilon_{y}(\lambda) \hat{y}+\epsilon_{z}(\lambda) \hat{z}$

with the components $\epsilon_{y}$ and $\epsilon_{z}$ given by:

$\epsilon_{j}(\lambda)=\frac{\iint j I(y, z, \lambda) \mathrm{d} y \mathrm{~d} z}{F(\lambda)} ; j=y, z$

where $\hat{y}$ and $\hat{z}$ are unit vectors. $F(\lambda)$ is the stellar spectral flux:

$F(\lambda)=\iint I(y, z, \lambda) \mathrm{d} y \mathrm{~d} z$ 
Integrations in Eqs. (6) and (7) are done over the visible stellar disc. $F(\lambda)$ is the zero order moment of the intensity map, while $\epsilon_{y}(\lambda) F(\lambda)$ and $\epsilon_{z}(\lambda) F(\lambda)$ are the first order moments.

For a given $\boldsymbol{B}_{\text {proj }}$ the measured $\phi$ includes information from both $\epsilon_{y}$ and $\epsilon_{z}$ (Eqs. (4) and (5)). The $y$ and $z$ components of $\boldsymbol{\epsilon}$ can, however, be separated by a vector projection of data obtained simultaneously in at least two distinct baseline position angles and/or by using the temporal evolution of the vector $\boldsymbol{\epsilon}$ resulting from Earth rotation (e.g. Jankov et al. 2001; Domiciano de Souza et al. 2003). In this paper we let $\xi$ free ( $\xi$ is the angle between the $y$ axis and $\boldsymbol{B}_{\text {proj }}$ as indicated in Fig. 1) and study separately the two perpendicular projections $\epsilon_{y}$ and $\epsilon_{z}$. A first order estimate of $\xi$, and thus of the orientation of the rotation axis on the sky plane, can be evaluated using the rigidrotator approximation. For example at $\xi=0^{\circ}$ one has $\boldsymbol{\epsilon}=\epsilon_{y} \hat{y}$ (maximum contribution of $\epsilon_{y}$ ) and conversely for $\xi=90^{\circ}$ one has $\boldsymbol{\epsilon}=\epsilon_{z} \hat{z}$ (maximum contribution of $\epsilon_{z}$, which is zero for rigid rotation). In the general case of a differentially rotating star we consider $\xi$ an unknown parameter as commented in Sect. 5.2.

\section{Differential rotation signatures on DI observables}

\subsection{Latitudinal differential rotation law}

Hereafter we consider the commonly used solar-like latitudinal angular differential rotation law:

$\Omega(l)=\Omega_{\mathrm{eq}}\left(1-\alpha \sin ^{2} l\right)=\frac{V_{\mathrm{eq}}}{R}\left(1-\alpha \sin ^{2} l\right)$

where $l$ is the latitude and $\Omega_{\mathrm{eq}}$ and $V_{\mathrm{eq}}$ are the angular and linear velocity at the equator, respectively. The differential rotation parameter $\alpha$ can be negative (acceleration of $\Omega$ towards the pole) or positive (acceleration of $\Omega$ towards the equator). Other laws than Eq. (8) could be chosen, but the main conclusions reached in this paper should not change significantly. Also, we assume that the star is spherical with linear radius $R$, with uniform surface gravity $(g)$ and effective temperature $\left(T_{\text {eff }}\right)$.

As interferometry measures angular sizes, all spatial coordinates are given in angular units, which requires one to the stellar distance $d$ to relate spatial and angular quantities. Thus, the linear rotational velocity projected onto the observer direction $\left(V_{\text {proj }}\right)$ is given by:

$V_{\text {proj }}(l)=[\boldsymbol{\Omega}(l) \otimes \boldsymbol{r}]_{x} d=-y d \Omega(l) \sin i$.

Thus, from Eqs. (8) and (9), $V_{\text {proj }}$ can be written as a function of the sky angular coordinates $y$ and $z$ of the reference frame of Fig. 1 as:

$V_{\text {proj }}(y, z)=-\frac{y}{\rho} V_{\text {eq }} \sin i\left[1-\alpha\left(\frac{z}{\rho} \sin i+\frac{x}{\rho} \cos i\right)^{2}\right]$

where $\rho=R d^{-1}$ is the stellar angular radius. Throughout this paper we adopt the sign convention according to which a positive velocity corresponds to displacements towards the observer. The $x$ coordinate can be replaced by the known relation $x=+\sqrt{\rho^{2}-y^{2}-z^{2}}$, for it corresponds to the visible stellar disc. Contrary to the case of rigid rotation $(\alpha=0)$, it follows
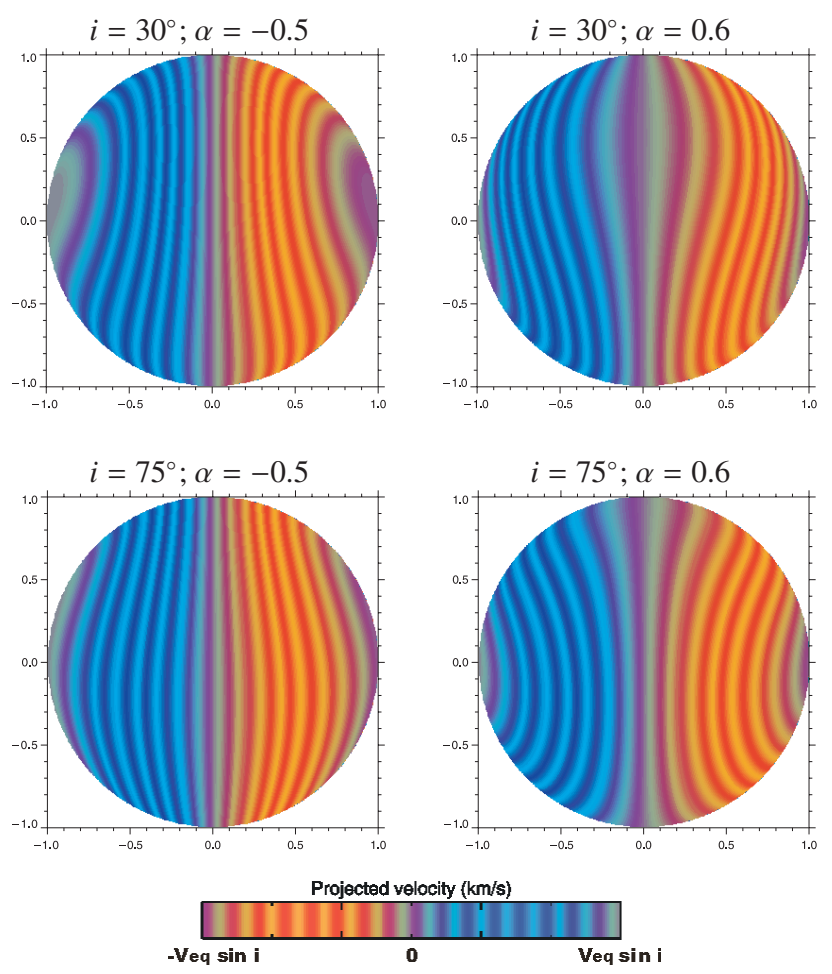

Fig. 2. Radial velocity maps for several combinations of $i$ and $\alpha$. A positive velocity corresponds to displacements towards the observer (blue shifts). For differential rotation, regions of constant projected velocity (equal velocity strips) are not straight vertical ones as is the case for rigid rotation $(\alpha=0)$. A direct consequence of these curved equal velocity strips is that the radial velocity maps become functions of the aspect angle $i$. Abscissas ( $y)$ and ordinates $(z)$ are given in units of stellar radius.

from Eq. (10) that the curves of constant radial velocity are not straight vertical lines, i.e., they are not defined by $y=$ const. Figure 2 shows 2D maps of $V_{\text {proj }}$ (hereafter called radial velocity map) for selected combinations of $i$ and $\alpha$. Note that the radial velocity maps do not depend on the specific value of $V_{\text {eq }}$, but only on $\alpha$ and $i$. To make clear the studied effects, Fig. 2 as well as most of the following graphical presentations are done for rather high $|\alpha|$, inspired by the values inferred by Stoeckley $\&$ Buscombe (1987). In Sect. 4.3, where the way of disentangling $\alpha$ and $i$ is presented, calculations are performed, however, for a wide range of $|\alpha|$ values beginning from $|\alpha|=0$.

\subsection{On the assumption of spherical objects}

No specific mechanism producing a differential rotation is studied in this work, nor do we intend to apply it for a specific type of objects. In principle they can be as different as stars and planets. We then also let free the surface rotational law sketched by Eq. (8) and leave the possibility of having extreme cases such as equatorially-accelerated zonal flows where $\alpha>0$, or hypothetically contracting objects with cylindrical-like local angular momentum conservation, corresponding to $\alpha<0$. Recently, Oláh et al. (2003) measured $\alpha<0$ in the RS CVn star UZ Lib.

For simplicity we assumed spherical objects. The present formulation precludes application to stars with geometrical 
deformations, like in rigid rotators where the concomitant gravitational darkening effect cannot be neglected. To set the upper limit on rotational velocities at which our method can still be used in the present form, let us assume that the differences between the values of polar and equatorial local effective temperatures induced by the rotation do not exceed $5 \%$, i.e. the average expected uncertainties in the current $T_{\text {eff }}$ determinations of stars. Using a Roche model for the stellar surface equipotential, this implies $T_{\text {polar }} / T_{\text {eq }} \simeq(1+0.5 \eta)^{1 / 2} \lesssim 1.05$, so that $\eta=\omega^{2}\left[R_{\text {eq }}(\omega) / R_{\mathrm{p}}\right]^{3} \lesssim 0.21(\omega=$ ratio of angular velocities $\Omega / \Omega_{\mathrm{c}}$ with $\Omega_{\mathrm{c}}$ the critical rate) and thus, $\omega \lesssim 0.66$ and $R_{\text {eq }} / R_{\mathrm{p}} \lesssim 1.10$. This sets the sought upper limit for the equatorial velocities at $V_{\mathrm{eq}} \lesssim 0.52 V_{\mathrm{c}} \simeq 180\left(M / R_{0}\right)^{1 / 2} \mathrm{~km} \mathrm{~s}^{-1}$ (both mass $M$ and the non deformed stellar radius $R_{0}$ are in solar units). Thus, as a reasonable upper limit of linear velocities at which our formulation can still be valid, in our numerical examples we adopted $V_{\text {eq }} \sin i=100 \mathrm{~km} \mathrm{~s}^{-1}$. We recall, however, that in fast rotators seen pole-on the deviation from sphericity of the sky-projected stellar disc is negligible. Also in this case, the whole observed hemisphere reflects an average effective temperature that is close to the $T_{\text {eff }}$ of the star at rest. The geometrical deformations and the gravitational darkening effect can however be taken into account and this will be done elsewhere. For the present contribution, our aim is only to sketch the basics of the method, for which these effects will unnecessarily burden its presentation.

\subsection{Numerical model}

To obtain the DI observables (Eqs. (6) and (7)) we need to calculate the specific intensity emerging from every visible point of the stellar disc as well as a rotation law that provides the wavelength Doppler shifts of the emerging line radiation. This is necessary in order to define completely the intensity map $I(y, z, \lambda)$.

Intensity maps $I$ have been obtained from the codes TLUSTY and SYNSPEC (Hubeny 1988; Hubeny \& Lanz 1995), which calculate the specific intensity emerging from each visible grid point. The stellar grid, with each corresponding physical parameter, is calculated with a modified version of the code BRUCE (Townsend 1997). To obtain the photocenters and line spectra, integrations in Eqs. (6) and (7) are performed numerically. This interferometry-oriented model also calculates the amplitudes of $\mathcal{W}$ and differential phases directly from the FT of the monochromatic intensity maps (Eq. (3); for details see Domiciano de Souza et al. 2002). Thus, $I$ is completely defined by a set of input parameters defining the stellar atmosphere, the required spectral range, and the rotation law. Throughout this paper we analyse the effects due to the differential rotation in the frame of a hot star and adopt as working stellar parameters $V_{\mathrm{eq}} \sin i=100 \mathrm{~km} \mathrm{~s}^{-1}, \log g=4.0$, $T_{\text {eff }}=20000 \mathrm{~K}$, and the line He I $\lambda 5876$. The He I $\lambda 5876$ line has several components and it is affected by Stark broadening (Dimitrijevic \& Sahal-Brechot 1990), which makes it asymmetric. Calculated line intensity profiles for several limb angles $\mu$ are shown in Fig. 3. In spite of the intrinsic asymmetry of the He I line the characteristics of the predicted DI observables

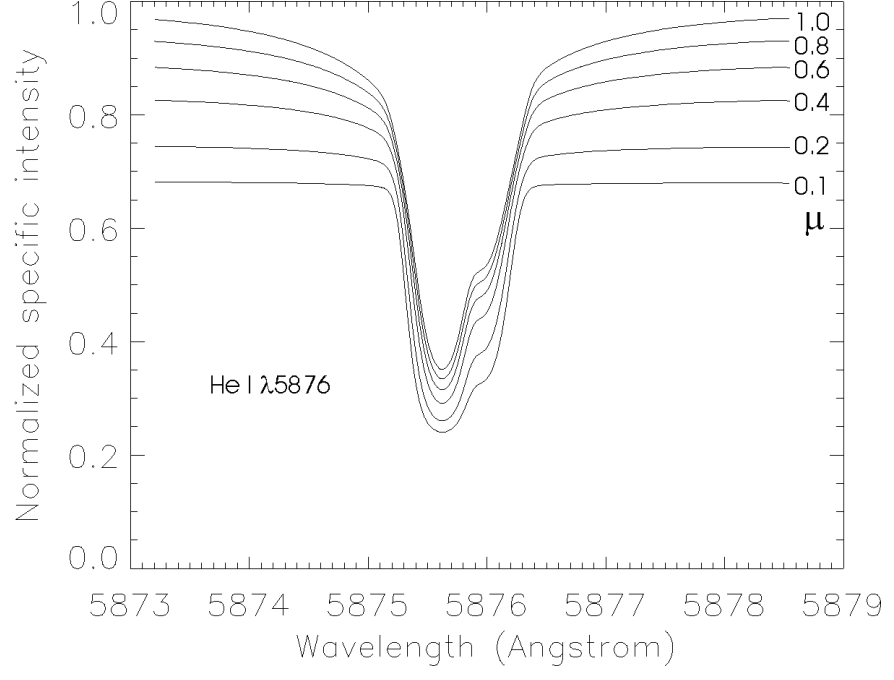

Fig. 3. Specific intensity $I(\lambda, y, z)=I(\lambda, \mu)$ of model He $\mathrm{I} \lambda 5876$ line profiles obtained for $\log g=4.0, T_{\text {eff }}=20000 \mathrm{~K}$ and $\mu=0.1$ to 1.0 . The line profiles are normalized to the local continuum intensity at $\mu=1.0$.

concerning differential rotation depend on the stellar differential rotation law but not on the intrinsic shape of the spectral line (Sect. 4.2). The photocenter displacements (Eq. (6)) are due to the differences in the length and shape of constant radial velocity curves as seen projected in the sky according to a given aspect angle.

Figure 4 (left) shows the DI observables for $i=45^{\circ}$, and $\alpha=+0.6$. The photocenters are given in units of stellar angular radius $\rho$, and the stellar flux is normalized to the continuum. To clarify the wavelength dependence of the photocenters, Fig. 4 (right) shows the intensity maps associated to the curves on the left at four selected wavelengths $\left(\lambda_{\mathrm{a}}, \lambda_{\mathrm{b}}, \lambda_{\mathrm{c}}\right.$ and $\left.\lambda_{\mathrm{d}}\right)$.

For $\lambda_{\mathrm{a}}$ and $\lambda_{\mathrm{b}}$ the photometric barycenter (filled circle) shifts towards the upper right quadrant of the stellar disc, i.e., both photocenter components are positive. The open circles indicate the geometrical center of the stellar visible disc. At $\lambda_{\mathrm{c}}$ the photometric barycenter shifts almost vertically towards the bottom $\left(\epsilon_{y}=0\right.$ and $\left.\epsilon_{z}<0\right)$. Finally, for $\lambda_{\mathrm{d}}$ the photometric barycenter shifts towards the upper left quadrant, so that $\epsilon_{y}<0$ and $\epsilon_{z}>0$.

\section{Parameter determination by Fourier analysis}

A powerful tool to derive information spectra and photocenters is the use of FT. For spectroscopic data these techniques were applied by many authors (cf. Gray 1977; Bruning 1981; Reiners \& Schmitt 2002, 2003). Here, we extend the FT method to DI and show how this combined technique can disentangle the differential rotation parameter $\alpha$ and the stellar inclination $i$.

\subsection{On the nature and choice of spectral lines}

The limb darkening across spectral lines can be more or less strongly dependent on the wavelength (Collins \& Truax 1995). Weak spectral lines with collision-dominated source functions 

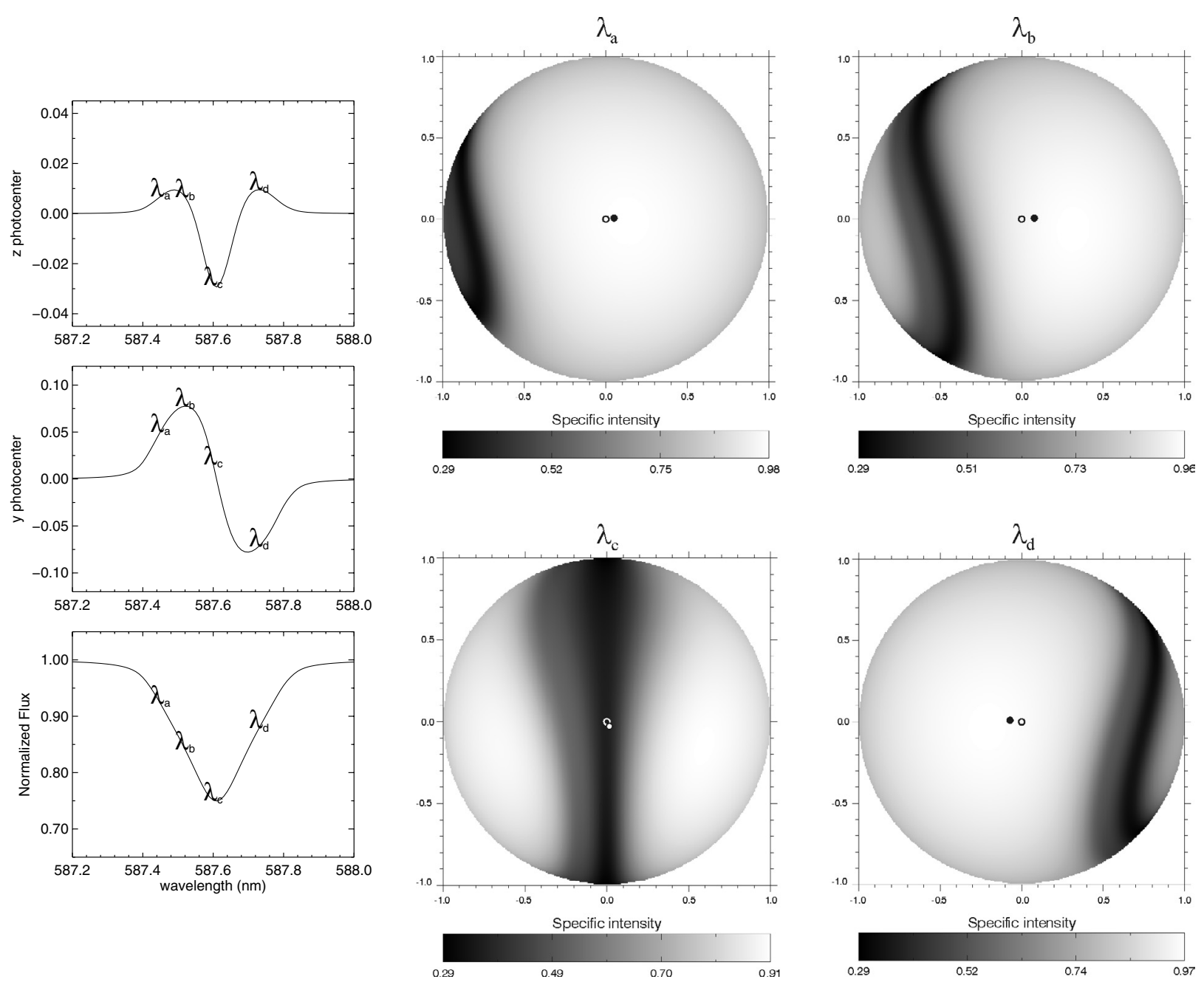

Fig. 4. Left: photocenter components, $\epsilon_{z}$ (top) and $\epsilon_{y}$ (middle), and the normalized spectral flux (bottom) across the asymmetric He I $\lambda 5876$ line. The calculations were performed for $V_{\mathrm{eq}} \sin i=100 \mathrm{~km} \mathrm{~s}^{-1}, \log g=4.0, T_{\mathrm{eff}}=20000 \mathrm{~K}, i=45^{\circ}$, and $\alpha=0.6$. Photocenter components are given in units of angular stellar radius $\rho$. The letters indicate selected wavelengths corresponding to a region in the blue line wing $\left(\lambda_{\mathrm{a}}\right)$, the highest $\epsilon_{y}$ value $\left(\lambda_{\mathrm{b}}\right)$, the central wavelength $\left(\lambda_{\mathrm{c}}\right)$, and the most positive value of $\epsilon_{z}\left(\lambda_{\mathrm{d}}\right)$. Right: intensity maps associated to the DI observables on the left side at the four selected wavelengths. The curved dark patterns correspond to Doppler shifts of the local line profile caused by differential rotation. These non-symmetrical intensity maps result in a displacement of the stellar photometric barycenter, i.e., the photocenter (filled circles), relative to the geometrical center (opened circles).

(Thomas 1965; Mihalas 1978) and affected as little as possible by pressure broadening effects (e.g. Stark) are expected to have limb darkening over the whole profile that is fairly the same as in the local continuum. The specific intensity of a spectral line can then be represented as a product of the fixed line profile and continuum where only the continuum intensity is affected by the limb darkening. On the other hand, strong lines with photoionization-dominated source functions (Thomas 1965; Mihalas 1978) and subject to broadenings like the Stark effect may have specific intensities in the cores that hardly show any dependence on the limb angle $\mu$, while their wings and the local continuum do vary strongly. In such cases, a marked dependence with wavelength of the limb darkening within the line is expected and the line intensity cannot be represented by a simple product of the constant line profile and the limb darkening-dependent continuum.

The limb darkening in the He I $\lambda 5876$ line profile calculated in Sect. 3.3 and represented as $I_{\lambda}(\mu) / I_{\lambda}(\mu=1)=1-\varepsilon_{\lambda}(1-\mu)$ is characterized by $\varepsilon_{\lambda}=0.24 \pm 0.01$ in the wings and in the core of the line, while $\varepsilon_{\lambda}=0.16 \pm 0.03$ in the line sides near the FWHM. As we noted in Sect. 3.3, the line undergoes large Stark broadening. We shall, however, use the following simple analytical representation of the intensity map for easy handling of the FT method and make more clear the advantages of its use:

$I(\lambda, y, z)=H\left(\lambda+\lambda_{0} \frac{V_{\text {proj }}(y, z)}{c}\right) I_{\mathrm{c}}(y, z)$.

This approximation assumes a fixed shape of the intrinsic line profile $H$ over the whole stellar disc. The specific intensity of the local continuum radiation $I_{\mathrm{c}}$, which includes limb darkening, is assumed wavelength independent over the narrow wavelength range of the studied spectral line.

Reiners \& Schmitt (2002) showed that the complete modelling of $H(\lambda)$ is in general not necessary to determine differential rotation, except for very slow rotators. Moreover, in 

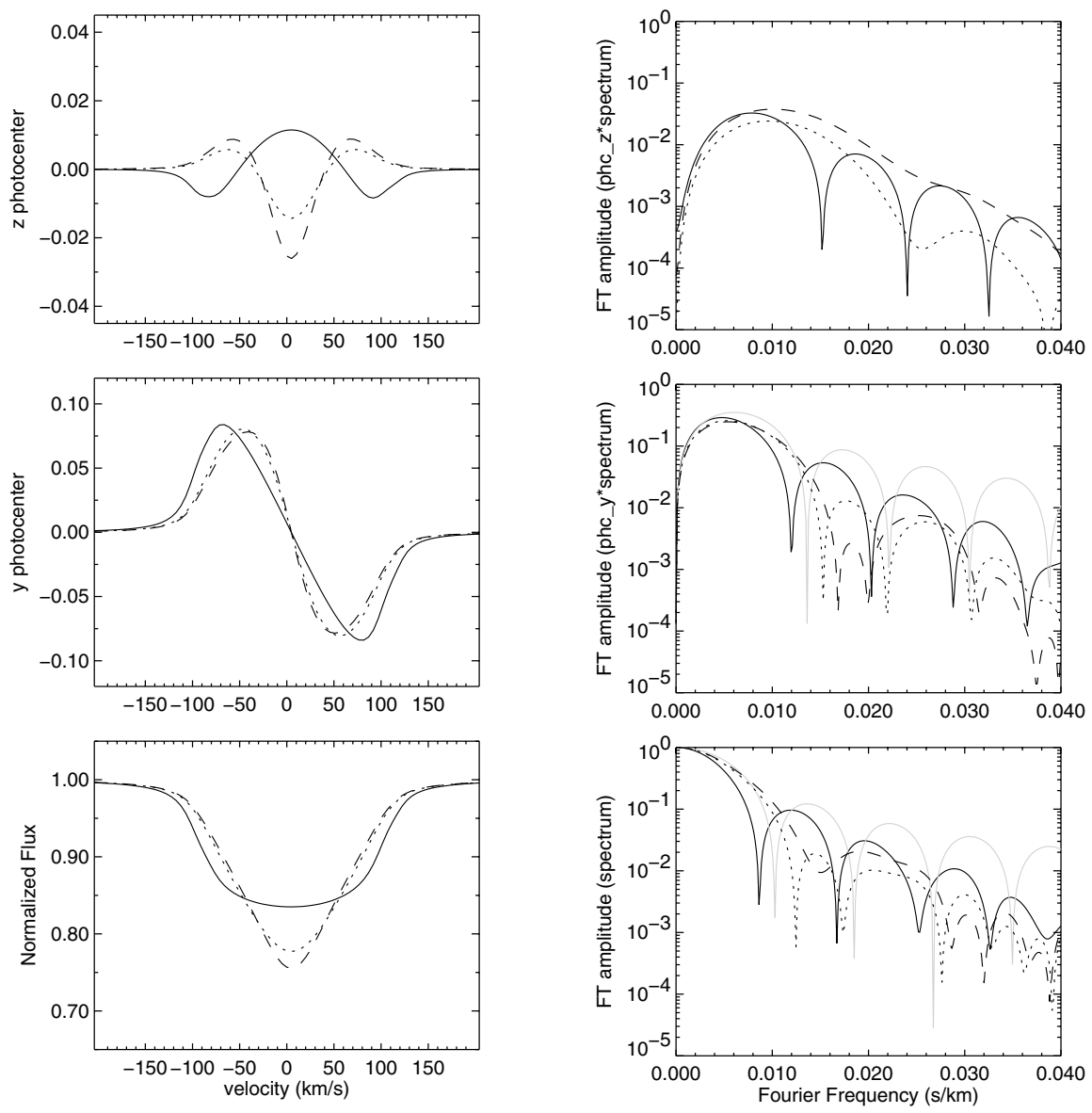

Fig. 5. Left: photocenter components, $\epsilon_{z}$ (top) and $\epsilon_{y}$ (middle), given in units of angular stellar radius. The line spectrum normalized to the local continuum flux is shown at the bottom. The calculations were performed for the asymmetric He I $\lambda 5876$ line, with $V_{\text {eq }} \sin i=100 \mathrm{~km} \mathrm{~s}{ }^{-1}$, $\log g=4.0, T_{\text {eff }}=20000 \mathrm{~K}$ and $i=45^{\circ}$. The curves correspond to a differential rotation parameter $\alpha$ equal to -0.5 (solid), 0.35 (dots) and 0.6 (dashes). Right: FT amplitudes of $F(\lambda), \epsilon_{y}(\lambda) F(\lambda)$, and $\epsilon_{z}(\lambda) F(\lambda)$ (Eq. (13)), normalized to the local continuum flux. Note that line styles correspond to the left curves and that they depend strongly on $\alpha$. The solid grey curves in the FT amplitudes of $F(\lambda)$ and $\epsilon_{y}(\lambda) F(\lambda)$ are the reference analytical Fourier transforms of the rotation broadening functions for uniform rotation and a linear limb darkening parameter $\varepsilon=0.23$ (see Appendix B). We recall that in the case of uniform rotation $\epsilon_{z}(\lambda)=0$, so that there is no grey curve in the upper right panel, i.e., $F T\left(\epsilon_{z}(\lambda) F(\lambda)\right)=0$.

Sect. 5.2 we shall compare the determination of $\alpha$ and $i$ using Eq. (11) with that obtained from detailed model line profiles calculated in Sect. 3.3 (see Fig. 3). This comparison will prove that the use of the simple and handy relation above (Eq. (11)) produces sensibly the same results as the use of detailed calculations.

\subsection{Differential rotation signatures in the Fourier domain}

According to the above approximations, it follows that the photocenters (Eq. (6)) and spectra (Eq. (7)) can be written as convolutions (for more details see Appendix A):

$\left(\begin{array}{l}F(\lambda) \\ \epsilon_{y}(\lambda) \\ \epsilon_{z}(\lambda)\end{array}\right)=\left(\begin{array}{c}1 \\ F^{-1}(\lambda) \\ F^{-1}(\lambda)\end{array}\right) H(\lambda) *\left(\begin{array}{l}G(\lambda) \\ G_{y}(\lambda) \\ G_{z}(\lambda)\end{array}\right)$

where $G, G_{y}$, and $G_{z}$ are the rotational broadening functions associated to $F(\lambda)$ and to the $(y, z)$-photocenter components, respectively. In Eq. (12) only the rotational broadening functions do carry information on global stellar parameters such as differential rotation, stellar inclination and limb darkening.

Applying the well known convolution theorem to the FT of the zero and first order moments of the intensity map, i.e., to $F(\lambda), \epsilon_{y}(\lambda) F(\lambda)$, and $\epsilon_{z}(\lambda) F(\lambda)$, we obtain:

$F T\left(\begin{array}{l}F(\lambda) \\ \epsilon_{y}(\lambda) F(\lambda) \\ \epsilon_{z}(\lambda) F(\lambda)\end{array}\right)=\widetilde{H}(\sigma) \times\left(\begin{array}{l}\widetilde{G}_{(j)}(\sigma) \\ \widetilde{G}_{y}(\sigma) \\ \widetilde{G}_{z}(\sigma)\end{array}\right)$

where $F T()$ is the Fourier transform operator and the tilded quantities represent the respective Fourier transforms. As for Eq. (12) in order to not burden this section with analytical developments, we display in Appendix B the expressions of the rotational broadening functions and their FT in the approximation of uniform rotation and linear limb darkening.

Figure 5 depicts the dependence of the DI observables on the differential rotation parameter $\alpha$ in the velocity (left) and in the Fourier (right) domains. Throughout this paper the Fourier frequencies were normalized so that the first zero position of 

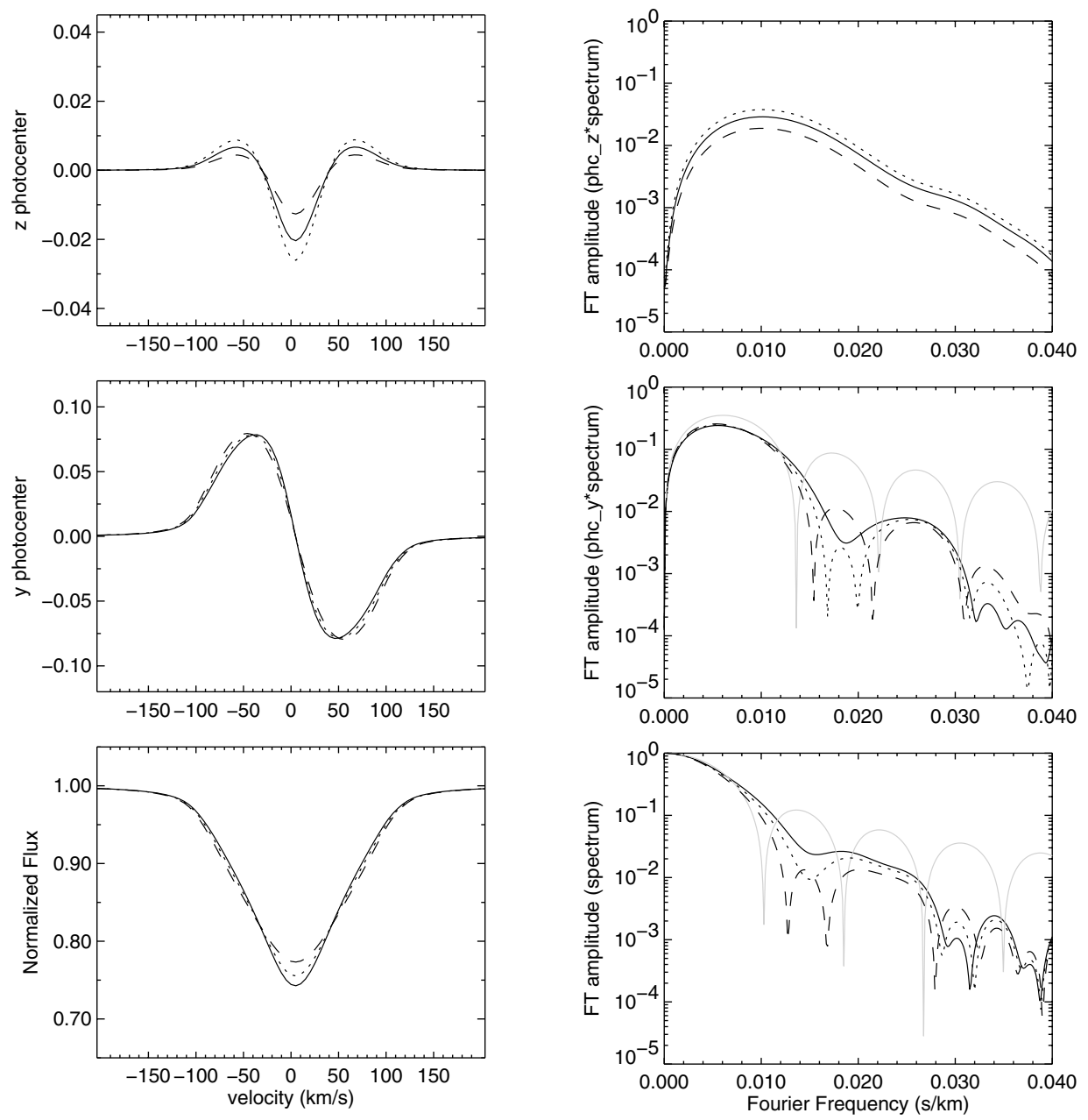

Fig. 6. Same as Fig. 5 but for $\alpha=0.60$. The curves correspond to $i$ equal to $25^{\circ}$ (solid), $45^{\circ}$ (dots) and $75^{\circ}$ (dashes).

a star with uniform rotation and no limb darkening is located at $\left(V_{\text {eq }} \sin i\right)^{-1}$, which is equal to $0.01 \mathrm{~s} / \mathrm{km}$ in our case. Note, in particular, that the sign of $\epsilon_{z}$ is inverted when we change from $\alpha<0$ to $\alpha>0$. The right column shows the respective FT amplitudes of $\epsilon_{z}(\lambda) F(\lambda), \epsilon_{y}(\lambda) F(\lambda)$ and $F(\lambda)$, normalized to the local continuum flux. In the abscissa we have the Fourier frequency $\sigma$, which is given in units of inverse velocity $(\mathrm{s} / \mathrm{km})$. Note that the positions of the FT zeros and the shape of lobes depend strongly on $\alpha$.

The dependence of the DI observables on $i$ for $\alpha=0.6$ is shown in Fig. 6, while Fig. 7 is for $\alpha=-0.50$. In both cases the inclination is $25^{\circ}$ (solid), $45^{\circ}$ (dots), and $75^{\circ}$ (dashes). These two figures show that all DI observables are sensitive to $i$, but not in the same way. The differential rotation signature on $\epsilon_{z}$ is the strongest for $i=45^{\circ}$, while it is of intermediate amplitude in $\epsilon_{y}$ and $F$. Moreover, for $\alpha>0$ the positions of zeros in the Fourier domain are highly dependent on $i$, resulting in secondary lobes shallowed or disappearing entirely in some cases, while for $\alpha<0$ this dependence is much weaker.

For the sake of a rapid comparison, in Figs. 5-7 we also show (grey curves) the analytical solutions for the FT of the rotational broadening functions derived in Appendix B $\left(\epsilon_{z}(\lambda)=0\right.$ and thus $\widetilde{G}_{z}=0$ at uniform rotation). These curves correspond to a linear limb darkening parameter $\varepsilon=0.23$, which is compatible with the stellar parameters adopted in our detailed numerical calculations. They decrease more slowly than those from our model because they are not multiplied by $\widetilde{H}(\sigma)($ Eq. (13)).

\subsection{Removal of $\mathrm{H}$ at all Fourier frequencies}

Thanks to the products in Eq. (13) one can find several receipts to obtain information on differential rotation independently from $H$.

To study a solar-like differential rotation law (Eq. (8)), Reiners \& Schmitt (2002) calculated the FT of line profiles $[\widetilde{F}(\sigma)=\widetilde{H}(\sigma) \widetilde{G}(\sigma)]$. Their study is based on the fact that the Fourier frequencies where $\widetilde{G}=0$ are independent from $\widetilde{H}$. Thus, in the usual situation where $G$ is sufficiently wider than $H$, the positions of zeros at low Fourier frequencies come only from $G$, i.e., they are only influenced by differential rotation, stellar inclination, and limb darkening. Reiners \& Schmitt (2002) showed that differential rotation can be distinguished from limb darkening by using the ratio between the second and the first zero positions of $\widetilde{F}$, which they denoted by $q_{2} / q_{1}$. However, they suffered from the difficulty that $i$ and $\alpha$ cannot be disentangled by studying only spectroscopic data. 

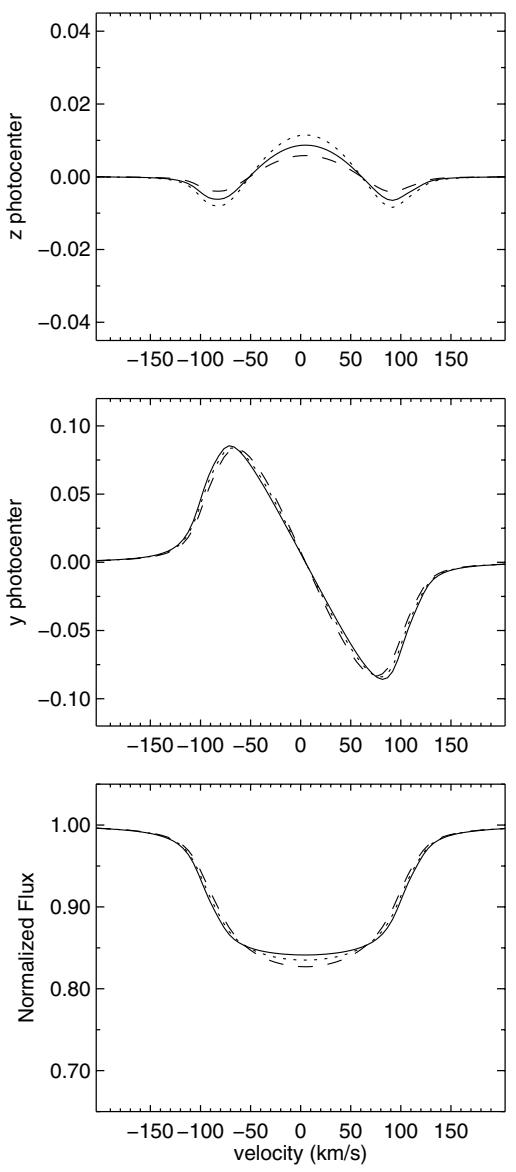

Fig. 7. Same as Fig. 6 but for $\alpha=-0.50$.

In the following, we show how the spectro-interferometry can separate $\alpha$ from $i$. Contrarily to other methods, based on the use of the zeros of $\widetilde{F}$, we can use all the available Fourier frequencies. of $H$ :

From Eq. (13) we define two new quantities independent

$R_{y}(\sigma) \equiv \frac{\left|F T\left(\epsilon_{y}(\lambda) F(\lambda)\right)\right|}{|F T(F(\lambda))|}=\frac{\left|\widetilde{G}_{y}(\sigma)\right|}{|\widetilde{G}(\sigma)|}$

and

$R_{z}(\sigma) \equiv \frac{\left|F T\left(\epsilon_{z}(\lambda) F(\lambda)\right)\right|}{|F T(F(\lambda))|}=\frac{\left|\widetilde{G}_{z}(\sigma)\right|}{|\widetilde{G}(\sigma)|}$

where the ratios $R_{y}(\sigma)$ and $R_{z}(\sigma)$ are valid for $\widetilde{G}(\sigma) \neq 0$. Clearly, these two quantities are independent from $H$ at all Fourier frequencies, contrary to the ratio $q_{2} / q_{1}$, which makes direct use of the zero positions of $\widetilde{F}$ only. Using the analytical expressions in Appendix B it is easy to derive $R_{y}$ for a given limb darkening law and a uniformly rotating spherical star. In this case it is $R_{z}=0$.

\subsection{Disentangling $\alpha$ and $i$}

The dependence of $R_{y}$ and $R_{z}$ on $\alpha$ and $i$ is shown as contour plots in Figs. 8 and 9, respectively. Hereafter we fix the Fourier frequency to $0.5 /\left(V_{\mathrm{eq}} \sin i\right)$ so that these contour plots can be
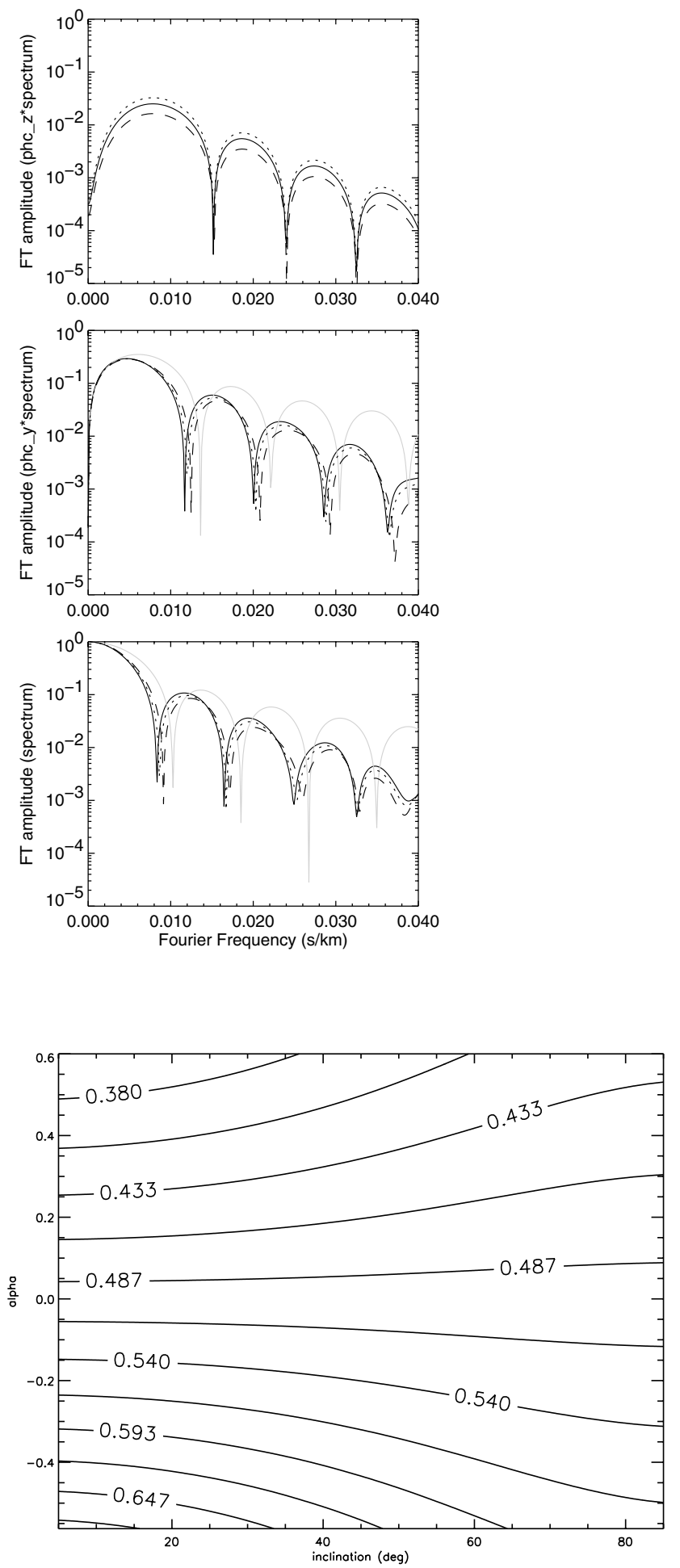

Fig. 8. Contour-plot of $R_{y}$ in the $i-\alpha$ plane for a Fourier frequency fixed to $0.5 /\left(V_{\mathrm{eq}} \sin i\right)$.

shown in the $i-\alpha$ plane. We note that Fig. 8 is qualitatively similar to Fig. 6 of Reiners \& Schmitt (2002), obtained for the spectroscopic quantity $q_{2} / q_{1}$. This is an expected result since $\epsilon_{y}$ contains spatial information similar to line spectra, i.e., perpendicular to the projection of the rotation axis onto the sky.

Thanks to the differences between the contour-plot patterns in Figs. 8 and 9 the parameters $\alpha$ and $i$ can be disentangled. 


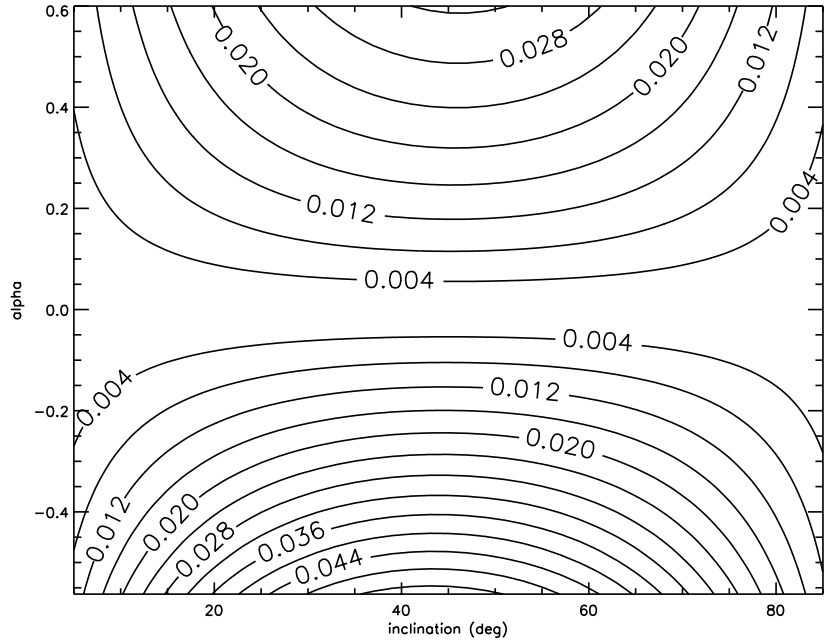

Fig. 9. Same as Fig. 8 but for $R_{z}$. Note that the dependence of $R_{z}$ on $\alpha$ and $i$ is significantly different from that of $R_{y}$ (Fig. 8), even though they are both independent from $H$.

To illustrate this possibility we show in Fig. 10 two examples of the intersection between $R_{y}$ (dashed curves) and $R_{z}$ (solid curves). Clearly, $\alpha$ and $i$ are much more constrained when $R_{y}$ and $R_{z}$ are considered simultaneously (black filled regions). To simulate a more realistic case, $R_{y}$ and $R_{z}$ are represented with a finite width corresponding to $1 \%$ and $5 \%$ uncertainties, respectively. As discussed in Sect. 5, uncertainties in the differential phases of the order of a few percents can be attained by modern interferometers. Moreover, we set the uncertainties in $R_{z}$ as 5 times higher than for $R_{y}$, in agreement with the relative signal amplitude of the photocenters $\epsilon_{y}$ and $\epsilon_{z}$.

It is apparent from Fig. 10 (top) that two (mathematical) separate parameter-space regions are possible. In many cases they identify two not highly different values of $\alpha$, but two distinct inclinations. The most probable parameter-space region can be identified for example from independent spectroscopic data that allow us to derive the ratio $Q=\alpha / \sqrt{\sin i}$ with an uncertainty $\Delta Q \lesssim 0.1$ (Reiners \& Schmitt 2003). If according to this $\Delta Q$ we can detect a statistically significant difference between the individual $Q$ derived from both solution regions, the sought couple $(\alpha, i)$ can be identified. When this separation is not possible, the average value from the $\alpha$ 's found can by itself represent a reliable estimate of $\alpha$ (as well as of its $\operatorname{sign}$ ) isolated from $\sin i$. On the other hand, knowing that the inclination angle of stars is the stellar unknown parameter par excellence, the fact that two possible values for it can be estimated opens numerous possibilities for consistency tests of stellar fundamental parameters. The simplest one implies finding the solution leading to $V_{\text {eq }} \sin i / \sin i<V_{\text {crit }}$. Other possibilities are given either by the time series analysis of stellar pulsations, as in hot stars (e.g. Levenhagen et al. 2003; Floquet et al. 2002; Janot-Pacheco et al. 1999), or by time variations in Ca II H and K emission-line fluxes in cold stars (e.g. Vaughan et al. 1981), which help to find the stellar rotational period, which combined with other known stellar fundamental parameters gives $V_{\text {eq }}$ and leads to infer the inclination which better represents the measured $V_{\text {eq }} \sin i$ and couple $(\alpha, i)$.
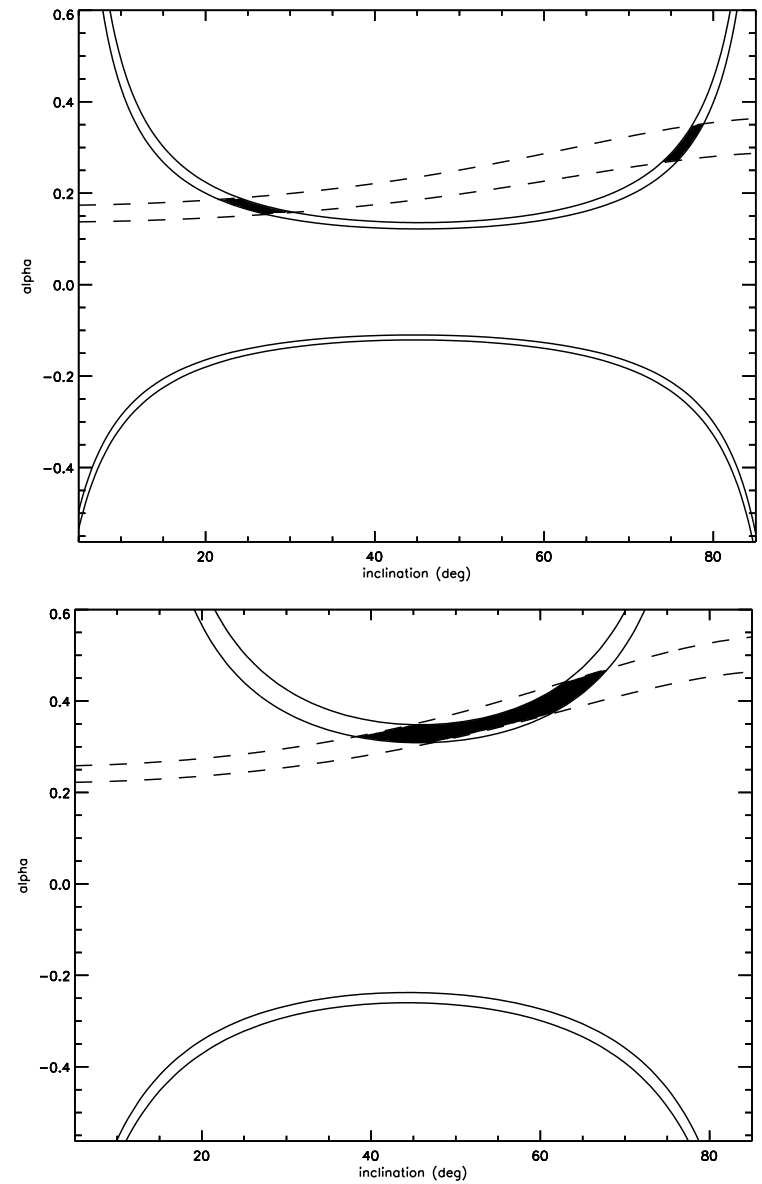

Fig. 10. Two examples of the intersection between $R_{y}$ (dashes) and $R_{z}$ (solid), from Figs. 8 and 9 respectively. The finite width for these quantities correspond to uncertainties of $1 \%$ for $R_{y}$ and $5 \%$ for $R_{z}$. These values are compatible with the expected uncertainties for modern interferometers and with the relative signal amplitude of the photocenters $\epsilon_{y}$ and $\epsilon_{z}$. Note that $\alpha$ and $i$ are well constrained only when $R_{y}$ and $R_{z}$ are considered simultaneously.

Figure 10 (bottom) is a different example where $R_{y}$ and $R_{z}$ result in a unique parameter-space region identified as solution for $(\alpha, i)$. We recall that although the examples shown in Figs. 8-10 correspond to a fixed Fourier frequency, our method is valid over the whole Fourier domain. Moreover, higher Fourier frequencies constrain better $\alpha$ and $i$. For example, at a Fourier frequency corresponding to $0.7 /\left(V_{\text {eq }} \sin i\right)$ the uncertainties in $\alpha$ and $i$ would be $\simeq 25 \%$ smaller if they are determined as in the upper panel of Fig. 10 and $\simeq 15 \%$ smaller if they correspond to intersections like on bottom panel of Fig. 10. In both kinds of intersections the regions coincide and have nearly the same barycenter.

\section{Discussion}

\subsection{Incidence on the results of detailed line profile calculations}

Figure 10 was obtained using a simplified representation of the limb angle-dependent He I $\lambda 5876$ line profile (Eq. (11)). The intersections of $R_{y}$ and $R_{z}$ curves were recalculated using the detailed $\mu$-dependent line profiles obtained in Sect. 3.3 


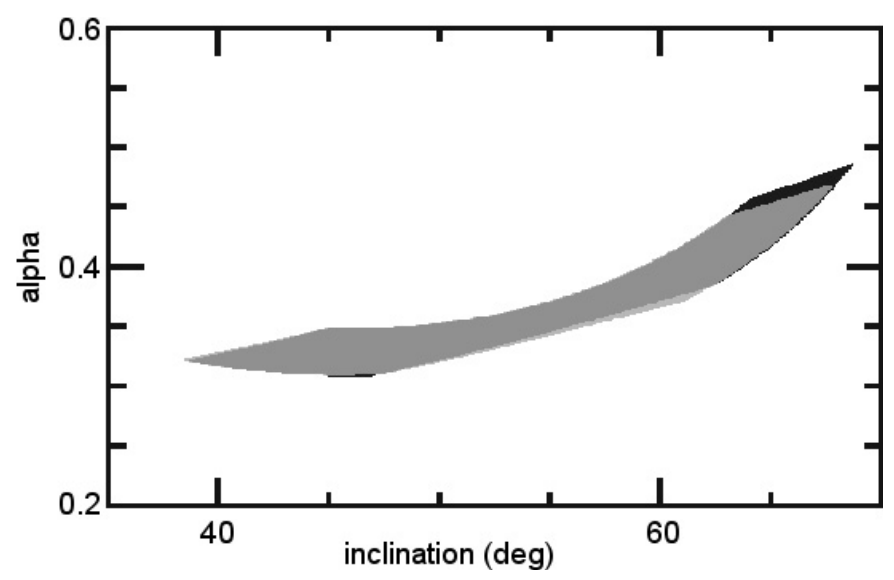

Fig. 11. Comparison between the expected $(\alpha, i)$ solution parameter space regions calculated using: (1) the approximation given by Eq. (11) which assumes a fixed shape of the local line profile with a wavelength independent limb darkening law (light gray zone), and (2) the detailed modelling of the He I $\lambda 5876$ line profile presented in Sect. 3.3 (black underlying zone). The strong resemblance between both solution regions (dark gray zone) proves the insensitivity of our method of $(\alpha, i)$ determination to a detailed local line profile treatment and thus, its robustness.

(Fig. 3). As before, the DI observables were evaluated numerically from Eqs. (6) and (7), i.e., without imposing any convolution between the local and rotation profiles. Figure 11 shows the solution zone thus obtained corresponding to the bottom panel of Fig. 10 where the higher differences appear. It can be seen, however, that both curvilinear quadrilaterals coincide almost perfectly and that they have virtually the same solution barycenter. This result shows that we can use Eq. (11) safely to represent lines like $\mathrm{He} \mathrm{I} \lambda 5876$ to set the bases of our $(\alpha, i)$ parameter determination method. Additionally, as noted in the preceding section, the further use of several Fourier frequencies can help to shrink the $(\alpha, i)$ solution quadrilateral domains.

\subsection{Further comments on the parameter determination}

For the He I $\lambda 5876$ line treated in the present paper not only have we used a linear approximation of the continuum intensity limb darkening, but also neglected its wavelength dependence within the line profile. For the $\left(T_{\text {eff }}, \log g\right)$ model atmosphere adopted, the linear limb darkening coefficient varies in the interval $0.10 \lesssim \varepsilon_{\lambda} \lesssim 0.25$. However, we showed that the $\lambda$-dependence of $\varepsilon$ in the line does not revamp our $(\alpha, i)$ parameter determination. Nevertheless, we can still ask whether the uncertainties in the stellar fundamental parameter determination may affect the parameter inference. To appreciate in a simple way the dependence of $R_{y}$ and $R_{z}$, let us assume Eq. (11) with the local continuum radiation $I_{\mathrm{c}}$ given by a linear limb darkening law as in Eq. (B.3) and the line profile $H$ given by a Gaussian function centered at $\lambda_{0}=5875.7 \AA$ :

$H_{\text {Gauss }}(\lambda)=1-H_{0} \exp \left[-\pi H_{0}^{2} \frac{\left(\lambda-\lambda_{0}\right) 2}{W 2}\right]$ where $H_{0}$ and $W$ are the central depths and the equivalent width, respectively. Figure 12 shows the DI observables in the velocity and Fourier domains for selected values of $\varepsilon, W$ (in $\AA$ ), and $H_{0}$, hereafter denoted by $\left(\varepsilon, W, H_{0}\right)$. The right column of Fig. 12 shows that the ratios $R_{y}$ and $R_{z}$ for all parameterized lines coincide over the whole studied frequency range, as expected from Eqs. (14) and (15). From the left column it is clear that the DI observables, notably the photocenter components, are sensitive to $\left(\varepsilon, W, H_{0}\right)$. This dependence is also seen in the Fourier domain (middle column). However, the parameters used were chosen freely without caring if they represent a given star or not. In actual cases, all parameters $\left(\varepsilon, W, H_{0}\right)$ can be determined in advance precisely using observed line profiles and/or model calculations for the adopted stellar fundamental parameters $\left(T_{\text {eff }}, \log g, V_{\text {eq }} \sin i\right)$, which may have uncertainties of at least up to $10 \%$. From our own model atmosphere calculations we find that the errors committed in the estimations of the continuum limb darkening coefficient range from $\Delta \varepsilon_{\mathrm{UV}} \simeq$ $-0.35\left(\Delta T_{\text {eff }} / T_{\text {eff }}\right)\left(\lambda_{\mathrm{UV}} \sim 1000 \AA\right)$ to $\Delta \varepsilon_{\mathrm{IR}} \simeq-0.05\left(\Delta T_{\mathrm{eff}} / T_{\mathrm{eff}}\right)$ $\left(\lambda_{\mathrm{IR}} \sim 2 \mu \mathrm{m}\right)$ in all temperatures $7000 \mathrm{~K}<T_{\text {eff }}<40000 \mathrm{~K}$ and gravities $2.0<\log g<5.0$, so that estimates of $T_{\text {eff }}$ to within $10 \%$ produce too small uncertainties in $\varepsilon$ to have any sensitive effect on $R_{y}$ and $R_{z}$. Errors for $\log g$ produce even smaller uncertainties in $\varepsilon$.

Let us also note that in the same ranges of temperature and gravity given above the linear approximation of the limb darkening law is appropriate for the directional cosinus $0.3 \lesssim \mu \lesssim 1.0$ interval. Deviations from linearity are for a stellar disc fraction $\Delta S / S=\mu_{L}^{2}\left(\mu_{L}=0.3\right)$ that contributes less than $6 \pm 1 \%$ of the total observed flux, which justifies the use of linear darkening laws.

From DI observations the whole set of unknown parameters of differential spherical rotators $\left(\alpha, i, V_{\mathrm{eq}}, \rho, \xi\right)$ can be derived. If the limb darkening coefficient is not estimated beforehand using model atmospheres, it can also be considered as a free parameter to be determined through the fit of the FT. Parameters like $\rho$ and $\xi$ are, in general, free parameters of the problem that are determined using the DI observations. However, the techniques relevant for that are not within the scope of the present paper.

The discussion of the determination of all these parameters for fast rotators will be done in a forthcoming contribution, where the gravitational darkening effect is also included.

\subsection{Instrumental requirements}

Although we would like to focus this paper only on the presentation of a new method of studying stellar surface differential rotation, let us also have a quick look at the contemporary interferometers to see whether they are sensitive enough for the present method to be exploited. Three main instrumental requirements should be fulfilled:

- spectral resolution $R$ high enough to result in at least a few $(\simeq 5)$ measurements across the spectral line profile;

- angular resolution $\left(R_{\mathrm{a}}=1.22 \lambda / B_{\mathrm{proj}}\right)$ of at least 0.1 of the stellar angular diameter $\oslash$; 

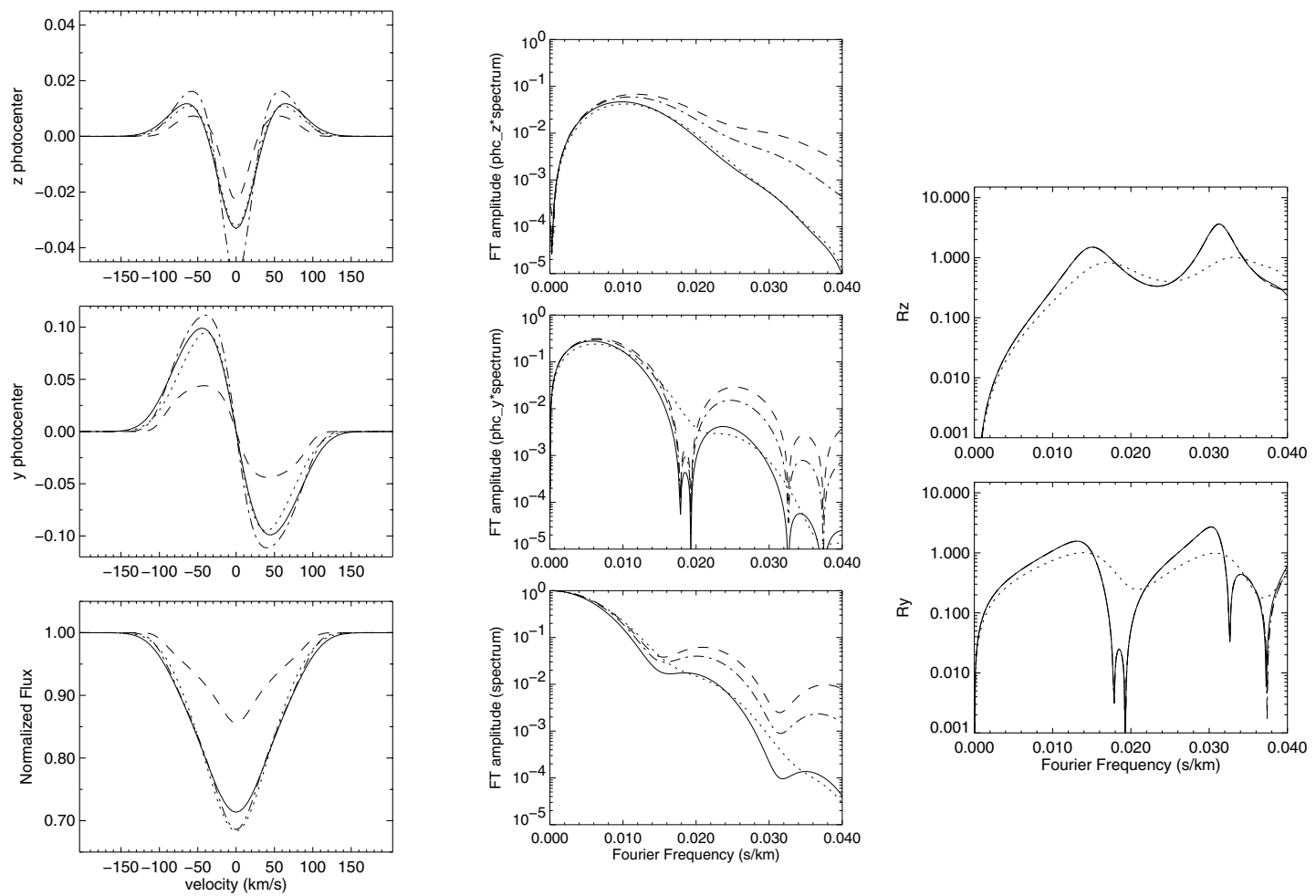

Fig. 12. Left column: photocenter components $\epsilon_{z}$ (top), $\epsilon_{y}$ (middle) (both given in units of the angular stellar radius) and the normalized line spectrum (bottom) for a linear limb darkening law (Eq. (B.3)) and a Gaussian local line profile (Eq. (16)). Calculations are done for selected values of parameters presented as $\left(\varepsilon, W, H_{0}\right)$. The results shown were obtained for $V_{\mathrm{eq}} \sin i=100 \mathrm{~km} \mathrm{~s}^{-1}, i=45^{\circ}$, and $\alpha=0.6$. Full-drawn curves correspond to $\left(\varepsilon, W, H_{0}\right)=(0.23,0.69,0.6)$, which represents a continuum radiation intensity similar to that used in the previous sections of this paper. The other curves are for parameters $(1.0,0.69,0.6)$ (dots), $(0.23,0.29,0.6)$ (dashes), and $(0.23,0.69,0.9)($ dot-dashes). Middle column: the corresponding FT amplitudes. Right column: ratios $R_{z}$ and $R_{y}$ for all curves. As expected from Eqs. (14) and (15), $R_{y}$ and $R_{z}$ do not depend on $W$ and $H_{0}$. However, they depend on $\varepsilon$ as is clear from the separate dotted curve $(\varepsilon=1.0)$.

- differential phases (Eq. (3)) measured with precisions high enough so that the uncertainty in the photocenter is $\lesssim 10^{-3}$ of the stellar angular radius $\rho(=0.50)$, i.e., $\$ 10 \%$ of the photocenter amplitudes expected for differential rotators.

In this context we consider now in more detail the AMBER instrument (Petrov et al. 2003; Malbet et al. 2003) to be soon installed on the VLT Interferometer (VLTI; Glindemann et al. 2003). VLTI disposes of up to 254 independent baselines with a maximum separation of $\sim 200 \mathrm{~m}$. AMBER operates in the $J, H$, and $K$ bands with a spectral resolution of 35,1000 , or 10000 . Uncertainties in the differential phases down to $\simeq 10^{-4} \mathrm{rad}$ can be obtained by VLTI/AMBER in its high precision mode. It can attain $R_{\mathrm{a}} \gtrsim 1.5$ marcsec (mas) for the longest baselines. We note that despite the fact that we have presented our method for $(\alpha, i)$ determination based on a test He I line in the visible spectrum range for a hot star, several spectral lines of different elements that depend on the stellar spectral type, with more or less similar intensity, can be found in the $J, H$, and $K$ bands. Moreover, in the future VLTI/AMBER is planned to operate also in the red end of the visible spectrum.

Figure 13 shows the photocenter uncertainties $\sigma_{\epsilon}$ as a function of the stellar apparent $K$ magnitude $\left(m_{K}\right)$ calculated for the VLTI/AMBER with the $1.8 \mathrm{~m}$ Auxiliary Telescopes (AT). Calculations were performed for stars with angular diameters $\oslash$ equal to a fraction of the angular resolution of the interferometer $\left(R_{\mathrm{a}} / n\right.$ with $\left.n=3,5,10\right)$. The solid curves correspond to $1 \mathrm{~h}$ exposure time while the dashed curve is for $0.5 \mathrm{~h}$. The curves were obtained for the highest AMBER spectral resolution $R=10000$, which is most probably the only one useful for differential rotation studies. For a direct comparison with the photocenter curves of this paper, $\sigma_{\epsilon}$ is given in units of angular radius $\rho$. The limit $\sigma_{\epsilon}=10^{-2} \rho$ was obtained using the $\epsilon_{z}$ amplitudes related to the model of differential rotation calculated in the present work where $\alpha$ ranges roughly from -0.6 to +0.6 . This value should not be considered as an absolute limit, but as a reliable threshold beyond which even the detection of differential rotation becomes very difficult.

For stars brighter than $m_{K}=3$ the uncertainty $\sigma_{\epsilon}$ is small enough for differential rotation studies. For stars with $m_{K}>3$ one can reduce $\sigma_{\epsilon}$ to values as small as $10^{-3} \rho$, simply by increasing $t_{\text {exp }}$ or, more interesting, by decreasing $n$ through observations with longer baselines and/or smaller wavelengths. Moreover, for the VLT $8.2 \mathrm{~m}$ Unit Telescopes (UT) $\sigma_{\epsilon}$ is reduced by a factor of $\simeq 5$, which places most curves of Fig. 13 well below the $10^{-2} \rho$ threshold.

\section{Conclusions and summary}

In this paper we investigated the use of DI for the study of stellar surface differential rotation. We showed that the 


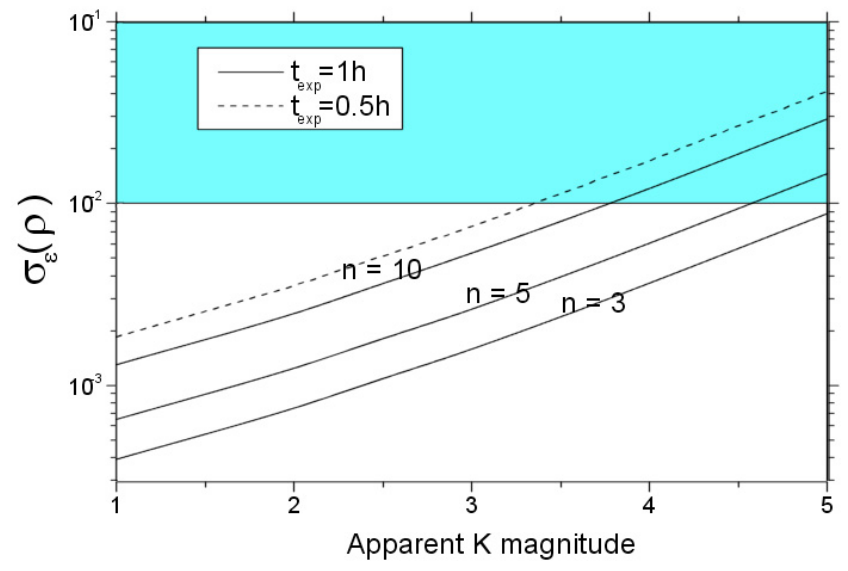

Fig. 13. Photocenter uncertainty $\sigma_{\epsilon}(\log$ scale) as a function of the stellar $K$ magnitude expected for observations at VLTI/AMBER with the $1.8 \mathrm{~m}$ Auxiliary Telescopes (AT). Calculations were performed for stars with angular diameters $\oslash$ equal to a fraction $(1 / n$ with $n=3,5,10)$ of the angular resolution of the interferometer $\left(R_{\mathrm{a}}=\right.$ $1.22 \lambda / B_{\text {proj }}$. For $n=10$ calculations were performed for two exposure times $t_{\exp }$ equal to 0.5 and $1 \mathrm{~h} . \sigma_{\epsilon}$ is given in units of angular radius $\rho(=0.5 \oslash)$. The $10^{-2} \rho$ limit is representative of uncertainties beyond which it becomes very difficult to measure differential rotation.

combined use of DI and the Fourier transform method is particularly suited for this purpose.

We showed that the DI observables, notably the photocenter component in the direction parallel to the projection of the stellar rotation axis onto the sky plane $\left(\epsilon_{z}\right)$, carry much more information on differential rotation and stellar inclination than do spectroscopic observations alone. Indeed, $\alpha$ (differential rotation rate) and $i$ (stellar inclination) can be disentangled from spectro-interferometric observations.

Additionally, our detailed numerical calculations, as well as those based on analytical representations of the radiation field, proved the great advantage of analyzing DI observables in the Fourier domain. The application of this Fourier method, represented by the quantities $R_{y}$ and $R_{z}$ defined in Sect. 4.3, totally removes the influence of the local line profile over all available Fourier frequencies. The inclusion of all these frequencies is an important advantage upon spectroscopy alone, and this allows a self consistent parameter determination to be performed.

In the appendices we present the needed reference analytical expressions related to the Fourier analysis of spectrointerferometric data. It is shown that the DI observables can be expressed as convolutions of the studied spectral line profiles with the corresponding rotational broadening functions. Although some of these equations were given in several earlier studies (e.g. Carroll 1933a,b; Böhm 1952; Lagarde 1994), here we use a different and homogeneous approach to derive them. To our knowledge, this is the first time that linear limb darkening is explicitly used in the equations related to the photocenters.

Finally, in the frame of differential rotation, rather high instrumental performances are needed to determine consistently the required free parameters. In particular, precise differential phase measures as well as high angular and spectral resolution are required. Our calculations demonstrated that foreseen interferometers, such as the VLTI/AMBER, will attain these instrumental requirements for realistic values of stellar magnitudes, angular diameters and exposure times.

Acknowledgements. A.D.S. acknowledges CAPES - Brazil (contract BEX 1661/98-1) for financial support. L.A. benefits from a postdoctoral position from CNES - France. We thank Dr. M. Vannier for the help with the calculations of the differential phase errors for VLTI/AMBER. Many thanks are due to the referee (Prof. Dr. N. Piskunov) for his constructive remarks which helped to improve the presentation of many issues in this paper.

\section{Appendix A: Line spectra and photocenters expressed as convolutions}

Whenever the shape of local line profile $H(\lambda)$ can be considered fixed over the whole observed stellar disc, we show here that the well known convolution relation for the line spectra is also valid for the photocenter. Let us consider an intensity map of the form (Eq. (11)):

$I(y, z, \lambda)=H\left(\lambda+\lambda_{0} \frac{V_{\text {proj }}(y, z)}{c}\right) I_{\mathrm{c}}(y, z)$

where $I_{\mathrm{c}}(y, z)$ is the specific intensity of the local continuum radiation assumed wavelength independent over the considered spectral range. It includes the limb darkening effect.

Defining the Doppler shift $\Delta \lambda$ as:

$\Delta \lambda=\Delta \lambda(y, z)=-\lambda_{0} \frac{V_{\text {proj }}(y, z)}{c}$

we can write the line spectrum $F$ (Eq. (7)) and the photocenter components $\epsilon_{y}$ and $\epsilon_{z}$ (Eq. (6)) in the compacted form:

$\left(\begin{array}{c}F(\lambda) \\ \epsilon_{y}(\lambda) \\ \epsilon_{z}(\lambda)\end{array}\right)=\left(\begin{array}{c}1 \\ F^{-1} \\ F^{-1}\end{array}\right) \iint\left(\begin{array}{c}1 \\ y \\ z\end{array}\right) H(\lambda-\Delta \lambda) I_{\mathrm{c}}(y, z) \mathrm{d} y \mathrm{~d} z$

where each line corresponds to an independent equation. In general, as $\Delta \lambda$ ranges from $\Delta \lambda_{\min }$ to $\Delta \lambda_{\max }, y$ ranges from $-\rho$ to $\rho$. In our case we have $\Delta \lambda_{\min }=-\Delta \lambda_{\max }$. Using Eq. (A.2) we can perform the coordinate change $y \rightarrow \Delta \lambda$ to obtain the following convolution relations:

$$
\begin{aligned}
\left(\begin{array}{l}
F(\lambda) \\
\epsilon_{y}(\lambda) \\
\epsilon_{z}(\lambda)
\end{array}\right) & =\left(\begin{array}{c}
1 \\
F^{-1} \\
F^{-1}
\end{array}\right) \int_{\Delta \lambda_{\min }}^{\Delta \lambda_{\max }} H(\lambda-\Delta \lambda)\left(\begin{array}{l}
G(\Delta \lambda) \\
G_{y}(\Delta \lambda) \\
G_{z}(\Delta \lambda)
\end{array}\right) \mathrm{d} \Delta \lambda \\
& =\left(\begin{array}{c}
1 \\
F^{-1} \\
F^{-1}
\end{array}\right) H(\lambda) *\left(\begin{array}{l}
G(\lambda) \\
G_{y}(\lambda) \\
G_{z}(\lambda)
\end{array}\right)
\end{aligned}
$$

where $G, G_{y}$, and $G_{z}$ are the rotational broadening functions associated to the spectra and the two photocenter components, respectively. We note that, while $H$ is just shifted by $\Delta \lambda$, the form of the rotational broadening functions depends on the adopted rotation law through Eq. (A.2), and on $I_{\mathrm{c}}$, since:

$\left(\begin{array}{l}G(\Delta \lambda) \\ G_{y}(\Delta \lambda) \\ G_{z}(\Delta \lambda)\end{array}\right)=\int_{f 1(\Delta \lambda)}^{f 2(\Delta \lambda)}\left(\begin{array}{l}1 \\ y \\ z\end{array}\right) I_{\mathrm{c}}(\Delta \lambda, z) \frac{\partial y}{\partial \Delta \lambda} \mathrm{d} z$ 
where $\partial y / \partial \Delta \lambda$ is the Jacobian corresponding to the coordinate change $y \rightarrow \Delta \lambda$. The convolution in Eq. (A.4) was deduced without any previous explicit knowledge of all terms involved in Eq. (A.5) that, in general, cannot be treated analytically for differential rotation laws.

\section{Appendix B: Analytical DI equations}

Here we apply the equations developed in Appendix A to the particular case of a uniformly rotating spherical star with linear limb darkening. For uniform rotation the linear rotation velocity projected onto the observer direction is given by Eq. (10) with $\alpha=0$ :

$V_{\text {proj }}(y)=-\frac{y}{\rho} V_{\text {eq }} \sin i$.

Thus, the regions of constant radial velocity are straight lines parallel to the projection of the rotation axis onto the sky, defined by $y=$ const. The coordinate change $y \rightarrow \Delta \lambda$ implies then:

$y=y(\Delta \lambda)=\rho \frac{\Delta \lambda}{\lambda_{0}} \frac{c}{V_{\mathrm{eq}} \sin i}=\rho \frac{\Delta \lambda}{\Delta \lambda_{\max }}$

where $\Delta \lambda_{\max }$ is the maximum rotational Doppler shift.

Additionally we consider that $I_{\mathrm{c}}(y, z)$ in Eq. (A.1) is given by a linear and wavelength independent limb darkening law:

$I_{\mathrm{c}}(y, z)=I_{0}(1-\varepsilon+\varepsilon \mu(y, z))$

where $\varepsilon$ is the limb darkening parameter, $I_{0}$ is the continuum intensity at the center of the visible stellar disc, and $\mu$ is the cosine of the angle between the normal to the surface at the point considered and the observer direction.

Under these assumptions we can thus rewrite the rotational broadening functions in Eq. (A.5) as:

$$
\left(\begin{array}{l}
G(\Delta \lambda) \\
G_{y}(\Delta \lambda) \\
G_{z}(\Delta \lambda)
\end{array}\right)=\int_{-\sqrt{\rho^{2}-y^{2}}}^{\sqrt{\rho^{2}-y^{2}}}\left(\begin{array}{l}
1 \\
y \\
z
\end{array}\right) I_{0}(1-\varepsilon+\varepsilon \mu) \frac{\rho}{\Delta \lambda_{\max }} \mathrm{d} z
$$

where $\rho$ is the stellar angular radius, $\varepsilon$ is the limb darkening parameter, $I_{0}$ is the continuum intensity at the center of the visible stellar disc, and $\mu$ is the cosine between the normal to the stellar surface and the observers direction at a given point. In the adopted reference frame (Fig. 1) $\mu$ is given by:

$\mu=\mu(y, z)=\frac{\sqrt{\rho^{2}-\left(y^{2}+z^{2}\right)}}{\rho}$.

We have then readily the known expression (e.g. Gray 1992, Chap. 17):

$$
\begin{aligned}
G(\Delta \lambda)= & I_{0} \frac{\rho^{2}}{\Delta \lambda_{\max }}\left\{2(1-\varepsilon) \sqrt{1-\left(\frac{\Delta \lambda}{\Delta \lambda_{\max }}\right)^{2}}\right. \\
& \left.+\frac{\pi}{2} \varepsilon\left[1-\left(\frac{\Delta \lambda}{\Delta \lambda_{\max }}\right)^{2}\right]\right\}
\end{aligned}
$$

obviously valid for $|\Delta \lambda| \leq \Delta \lambda_{\max }$. Contrary to the known rotation profile, Eq. (B.6) has not been normalized to the continuum flux:

$$
F_{\mathrm{c}}=I_{0} 2 \pi \rho^{2}\left(1-\frac{\varepsilon}{3}\right)^{-1}
$$

The rotation profile $G_{y}$ associated to the photocenter component $\epsilon_{y}$ is calculated in the same way as $G$, since the variable $y$ can be taken out of the integral over $z$ (Eq. (B.4)). We have thus $G_{y}$ proportional to $G$ :

$$
G_{y}(\Delta \lambda)=y(\Delta \lambda) G(\Delta \lambda)=\rho \frac{\Delta \lambda}{\Delta \lambda_{\max }} G(\Delta \lambda) .
$$

Finally, for the function $G_{z}$ associated to the photocenter component $\epsilon_{z}$ we have:

$G_{z}(\Delta \lambda)=0$

for the integral over $z$ is performed on an odd function over a symmetrical interval. This zero photocenter displacement in the $z$ direction is the consequence of the straight loci of points related to the radial velocity field.

Through a Fourier transform of Eq. (A.4) the contribution of the local line profile $H$ can be easily separated from the rotational profiles using the convolution theorem, which allow us to write the product:

$$
F T\left(\begin{array}{l}
F(\lambda) \\
\epsilon_{y}(\lambda) F(\lambda) \\
\epsilon_{z}(\lambda) F(\lambda)
\end{array}\right)=\widetilde{H}(\sigma) \times\left(\begin{array}{l}
\widetilde{G}(\sigma) \\
\widetilde{G}_{y}(\sigma) \\
\widetilde{G}_{z}(\sigma)
\end{array}\right)
$$

where $F T()$ is the Fourier transform operator and the tilde symbol represents the Fourier transform of the corresponding quantity; $\sigma$ is the Fourier variable corresponding to $\lambda$. Astrophysical information on the stellar surface is contained in the rotational broadening functions only. We can now derive analytical expressions for the Fourier transforms of the rotational profiles for the case of uniform rotation.

Since $G$ is an even function (Eq. (B.6)) and $G_{y}$ is odd (Eq. (B.8)), their corresponding Fourier transforms can be written as:

$\widetilde{G}(\sigma)=2 \int_{0}^{\Delta \lambda_{\max }} G(\lambda) \cos (2 \pi \lambda \sigma) \mathrm{d} \lambda$

and

$$
\begin{aligned}
\widetilde{G_{y}}(\sigma) & =\imath 2 \int_{0}^{\Delta \lambda_{\max }} G_{y}(\lambda) \sin (2 \pi \lambda \sigma) \mathrm{d} \lambda \\
& =\frac{\imath 2 \rho}{\Delta \lambda_{\max }} \int_{0}^{\Delta \lambda_{\max }} \lambda G(\lambda) \sin (2 \pi \lambda \sigma) \mathrm{d} \lambda
\end{aligned}
$$

where we adopted the integration variable $\lambda$ instead of $\Delta \lambda$. Note that $\widetilde{G}$ is real and that $\widetilde{G}_{y}$ is imaginary $(l=\sqrt{-1})$. The expressions given by Eqs. (B.11) and (B.12) can be rewritten using 
the Bessel functions of half integer order $\left(J_{ \pm \frac{1}{2}}\right)^{1}$, so that:

$\widetilde{G}(\sigma)=(2 \pi)^{\frac{1}{2}} \int_{0}^{\Delta \lambda_{\max }} G(\lambda)(2 \pi \lambda \sigma)^{\frac{1}{2}} J_{-\frac{1}{2}}(2 \pi \lambda \sigma) \mathrm{d} \lambda$

and

$$
\widetilde{G}_{y}(\sigma)=\frac{l(2 \pi)^{\frac{1}{2}} \rho}{\Delta \lambda_{\max }} \int_{0}^{\Delta \lambda_{\max }} \lambda G(\lambda)(2 \pi \lambda \sigma)^{\frac{1}{2}} J_{\frac{1}{2}}(2 \pi \lambda \sigma) \mathrm{d} \lambda .
$$

Integrals (B.13) and (B.14) can be evaluated analytically using the following relation (Gradshteyn \& Ryzhik 1965):

$$
\int_{0}^{1} x^{v+1}\left(1-x^{2}\right)^{\mu} J_{v}(y x) \mathrm{d} x=\frac{2^{\mu} \Gamma(\mu+1)}{y^{\mu+1}} J_{v+\mu+1}(y)
$$

where $\Gamma$ is the gamma function. Thus, the Fourier transforms of the rotation profiles $G$ and $G_{y}$, normalized to the continuum flux $F_{c}$, are given by:

$$
\begin{aligned}
& \frac{\widetilde{G}(\eta)}{F_{c}}=\frac{2}{\left(1-\frac{\varepsilon}{3}\right)}\left[(1-\varepsilon) \frac{J_{1}(\eta)}{\eta}+\varepsilon\left(\frac{\pi \eta}{2}\right)^{\frac{1}{2}} \frac{J_{\frac{3}{2}}(\eta)}{\eta^{2}}\right] \\
& \frac{\widetilde{G}_{y}(\eta)}{F_{c}}=\frac{l 2 \rho}{\left(1-\frac{\varepsilon}{3}\right)}\left[(1-\varepsilon) \frac{J_{2}(\eta)}{\eta}+\varepsilon\left(\frac{\pi \eta}{2}\right)^{\frac{1}{2}} \frac{J_{\frac{5}{2}}(\eta)}{\eta^{2}}\right]
\end{aligned}
$$

where $\eta=2 \pi \sigma \Delta \lambda_{\max } . J_{1}, J_{2}, J_{\frac{3}{2}}$ and $J_{\frac{5}{2}}$ are Bessel functions of the first kind and of integer and half-integer ${ }^{2}$ order. Note that $\widetilde{G}_{y}(\eta) / F_{c}$ is proportional to the stellar angular radius $\rho$. This is a super-resolution effect from differential interferometry, which allows $\rho$ to be determined even when the baseline is not long enough to resolve the star (further details in Chelli \& Petrov 1995a,b).

We note that Eq. (B.16) is given by Carroll (1933a,b) for $\varepsilon=0.6$, which can be used for grey atmospheres, and by Böhm (1952) for arbitrary $\varepsilon$. Equation (B.17) is given by Lagarde (1994) for $\varepsilon=0$, which is suitable for wavelengths around the core of strong absorption lines. However, the formalism adopted here allows a homogeneous and different way to deduce all these DI equations. Additionally, we introduce for the first time the linear limb darkening parameter in the equations relative to $\epsilon_{y}$.

\section{References}

Beckers, J. M. 1982, Opt. Acta, 29, 361

Böhm, K. 1952, Z. Astrophys., 30, 117

Bruning, D. H. 1981, ApJ, 248, 274

Chelli, A., \& Petrov, R. G. 1995a, A\&AS, 109, 389

Chelli, A., \& Petrov, R. G. 1995b, A\&AS, 109, 401
Carroll, J. A. 1933a, MNRAS, 93, 478

Carroll, J. A. 1933b, MNRAS, 93, 680

Collins II, G. W., \& Truax, R. J. 1995, ApJ, 439, 860

Diaz-Cordoves, J., Claret, A., \& Gimenez, A. 1995, A\&AS, 110, 329

Cranmer, S. R., \& Collins, G. W. 1993, ApJ, 412, 720

Dimitrijevic, M. S., \& Sahal-Brechot, S. 1990, A\&AS, 82, 519

Domiciano de Souza, A., Kervella, P., Jankov, S., et al. 2003, A\&A, 407, L47

Domiciano de Souza, A., Vakili, F., Jankov, S., Janot-Pacheco, E., \& Abe, L. 2002, A\&A, 393, 345

Floquet, M., Neiner, C., Jannot-Pacheco, E., et al. 2002, A\&A, 394, 137

Gies, D. R., \& Kullavanijaya, A. 1988, ApJ, 326, 813

Glindemann, A., Algomedo, J., Amestica, R., et al. 2003, Proc. SPIE, 4838,89

Gradshteyn, I. S., \& Ryzhik, I. M. 1965, in Table of integrals, series and products (San Diego: Academic Press)

Gray, D. F. 1973, ApJ, 184, 461

Gray, D. F. 1975, ApJ, 202, 148

Gray, D. F. 1977, ApJ, 211, 198

Gray, D. F. 1982, ApJ, 258, 201

Huang, S., \& Struve, O. 1953, ApJ, 58, 216

Hubeny, I. 1988, Comp. Phys. Comm., 52, 103

Hubeny, I., \& Lanz, T. 1995, ApJ, 439, 875

Jankov, S., Vakili, F., Domiciano de Souza Jr., A., \& Janot-Pacheco, E. 2001, A\&A, 377, 721

Janot-Pacheco, E., Jankov, S., Leister, N. V., Hubert, A. M., \& Floquet, M. 1999, A\&AS, 137, 407

Labeyrie, A. 1970, A\&A, 6, 85

Lagarde, S. 1994, Ph.D. Thesis, Dept. d'Astrophysique de l'UNSA, Nice, France

Levenhagen, R. S., Leister, N. V., Zorec, J., et al. 2003, A\&A, 400, 599

Malbet, F., Bloecker, Th., Foy, R., et al. 2003, Proc. SPIE, 4838, 917

Mihalas, D. 1978, Stellar Atmospheres, Second edition (San Francisco: Freeman and Company)

Oláh, K., Jurcsik, J., \& Strassmeier, K. G. 2003, A\&A, 410, 685

Petrov, R. G., Lagarde, S., \& N'Guyen Van Ky, M. 1995, in Stellar Surface Structure, ed. K. G. Strassmeier, \& J. L. Linsky, ASP Proc. IAU Symp., 176, 181

Petrov, R. G., Malbet, F., Weigelt, G., et al. 2003, Proc. SPIE, 4838, 924

Reiners, A., \& Schmitt, J. H. M. M. 2002, A\&A, 384, 155

Reiners, A., \& Schmitt, J. H. M. M. 2003, A\&A, 398, 647

Stoeckley, T. R., \& Buscombe, W. 1987, MNRAS, 227, 801

Thomas, R. N. 1965, Some Aspects of Non-Equilibrium Thermodynamics in the Presence of a Radiation Field (Boulder: Univ. of Colorado Press)

Townsend, R. H. D. 1997, MNRAS, 284, 839

Vakili, F., Mourard, D., Bonneau, D., Morand, F., \& Stee, P. 1997, A\&A, 323, 183

Vaughan, A. H., Preston, G. W., Baliunas, S. L., et al. 1981, ApJ, 250, 276

Wade, R. A., \& Rucinski, S. M. 1985, A\&AS, 60, 471

Zorec, J. 1994, Pulsation; Rotation; and Mass Loss in Early-Type Stars, IAU Symp., 162, 57

$$
\begin{aligned}
& { }_{1} J_{\frac{1}{2}}(\eta)=\left(\frac{2}{\pi \eta}\right)^{\frac{1}{2}} \sin (\eta) \text { and } J_{-\frac{1}{2}}(\eta)=\left(\frac{2}{\pi \eta}\right)^{\frac{1}{2}} \cos (\eta) . \\
& 2 J_{\frac{3}{2}}(\eta)=\left(\frac{2}{\pi \eta}\right)^{\frac{1}{2}}\left(\frac{\sin (\eta)}{\eta}-\cos (\eta)\right) \text { and } \\
& J_{\frac{5}{2}}(\eta)=\left(\frac{2}{\pi \eta}\right)^{\frac{1}{2}}\left[\left(\frac{3}{\eta^{2}}-1\right) \sin (\eta)-\frac{3}{\eta} \cos (\eta)\right] .
\end{aligned}
$$

